\title{
Descriptive Proteome Analysis to Investigate Context-Dependent Treatment Responses to OXPHOS Inhibition in Colon Carcinoma Cells Grown as Monolayer and Multicellular Tumor Spheroids
}

Julia Steinmetz, Wojciech Senkowski, Johan Lengqvist, Jenny Rubin, Elena Ossipova, Stephanie Herman, Rolf Larsson, Per-Johan Jakobsson, Mårten Fryknäs, and Kim Kultima*

Cite This: ACS Omega 2020, 5, 17242-17254

Read Online

ABSTRACT: We have previously identified selective upregulation of the mevalonate pathway genes upon inhibition of oxidative phosphorylation (OXPHOS) in quiescent cancer cells. Using mass spectrometry-based proteomics, we here investigated whether these responses are corroborated on the protein level and whether proteomics could yield unique insights into context-dependent biology. HCT116 colon carcinoma cells were cultured as monolayer cultures, proliferative multicellular tumor spheroids (P-MCTS), or quiescent (Q-MCTS) multicellular tumor spheroids and exposed to OXPHOS inhibitors: nitazoxanide, FCCP, oligomycin, and salinomycin or the HMG-CoAreductase inhibitor simvastatin at two different doses for 6 and $24 \mathrm{~h}$. Samples were processed using an in-depth bottom-up proteomics workflow resulting in a total of 9286 identified protein groups. Gene
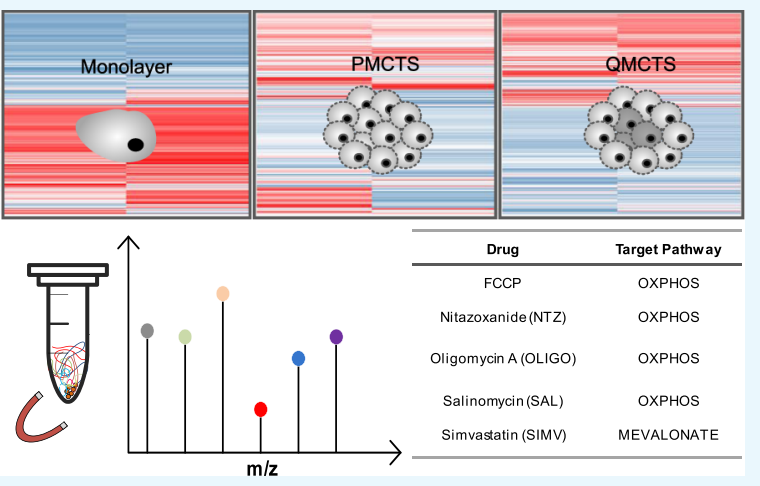
set enrichment analysis showed profound differences between the three cell systems and confirmed differential enrichment of hypoxia, OXPHOS, and cell cycle progression-related protein responses in P-MCTS and Q-MCTS. Treatment experiments showed that the observed drug-induced alterations in gene expression of metabolically challenged cells are not translated directly to the protein level, but the results reaffirmed OXPHOS as a selective vulnerability of quiescent cancer cells. This work provides rationale for the use of deep proteome profiling to identify context-dependent treatment responses and encourages further studies investigating metabolic processes that could be co-targeted together with OXPHOS to eradicate quiescent cancer cells.

\section{INTRODUCTION}

Cancer cells are usually characterized by their increased proliferation, resistance to apoptosis, invasiveness, and poor differentiation. However, it has been recently described that many solid tumors harbor nonproliferative, quiescent cells, residing in nutrient-deprived and hypoxic microenvironments, characterized by increased DNA damage and altered metabolism. ${ }^{1,2}$ This heterogeneous tumor microenvironment poses a therapeutic challenge, as chemo- and radiation therapy has been demonstrated to be less effective against cells in the poorly vascularized hypoxic niches, the presence of which has been associated with tumor relapse and poor prognosis. ${ }^{3}$ Thus, except targeting fast-growing tumor cells, there is also a need to find therapeutic strategies aimed at the quiescent tumor regions.

Three-dimensional (3D) cell cultures, in contrast to monolayer cultures, offer the possibility to investigate cell signaling, growth characteristics, and drug response in more in vivo like settings. Of various $3 \mathrm{D}$ culture types, multicellular tumor spheroids (MCTS) and tumor organoids have gained the most attention. In general, MCTS are usually generated from cell lines while tumor organoids are formed from primary cancer cells. Organoid cultures, which are grown in an external protein matrices (such as Matrigel), more accurately recapitulate the genetic and morphological characteristics of a primary tumor. However, because of the cost of organoid development and expansion and limited cellular material availability, MCTS can more easily and reproducibly be used in the large experimental setup, for example, high-throughput drug screening, ${ }^{4-6}$ and have been a valuable model for the studies of clinically relevant aspects of cancer biology, as they resemble morphological, functional, and microenvironmental features of in vivo tumor tissues. ${ }^{7}$ However, most commonly used spheroid models are highly proliferative MCTS (PMCTS), as they are usually maintained in standard nutrientrich culture media. P-MCTS experience high nutrient

Received: March 30, 2020

Accepted: June 22, 2020

Published: July 6, 2020 

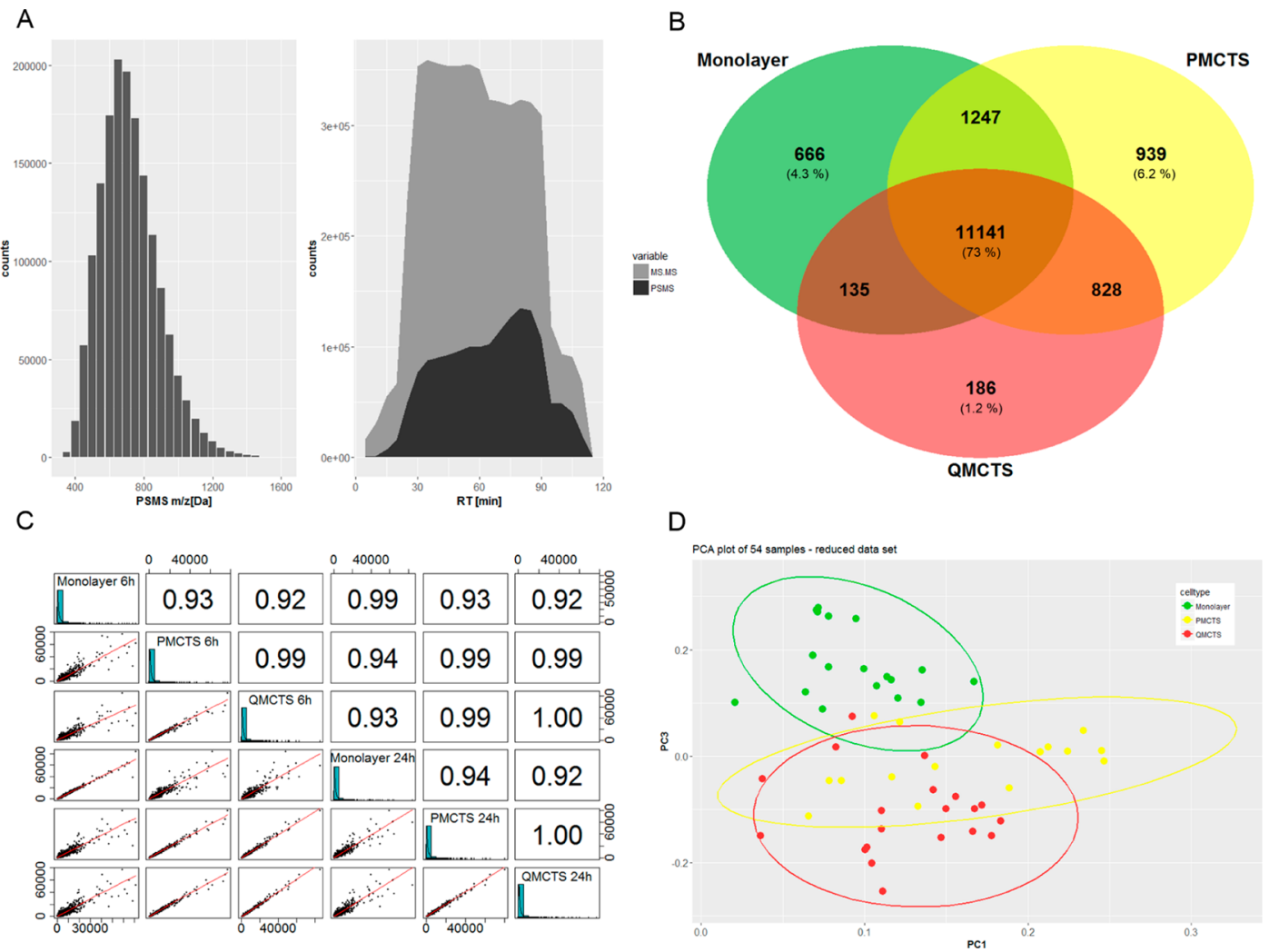

Figure 1. Quality control analysis and data assessment of bottom-up quantitative proteomics. (A) Mass range coverage and distribution of the obtained MS/MS spectrum information (5174157) and resulting PSMS (1594878) throughout the gradient. (B) Venn diagram of the whole data set comparing the proteome of the three cell types (including isoforms). Proteins present across $60 \%$ of the samples for each cell type were included in the analysis. (C) Reproducibility of the measured absolute protein abundances for the control set data is presented, where each row/ column depicts a control (DMSO treated) sample (Pearson correlation coefficient $>0.9$ ). (D) PCA (PC1, PC3) of quantified proteins throughout the whole data set after removal of outliers.

concentrations as the model contains a mixture of proliferating cells in the outer layers and quiescent cells toward the center of the spheroid. Recently, we have demonstrated that quiescent spheroids (Q-MCTS), cultured under in vivo-like conditions mimicking solid tumor microenvironments (i.e., nutrientdeprived and with low extracellular $\mathrm{pH}$ ), contain a larger fraction of quiescent cells and are therefore profoundly different from P-MCTS and monolayer cultures. In consequence, Q-MCTS enable modeling of quiescent regions of solid tumors and identification of unforeseen vulnerabilities of quiescent cancer cells. ${ }^{8,9}$ Furthermore, Q-MCTS allow studying drug responses of quiescent cancer cells in clearer detail, without the results being too obscured by the contribution from proliferating cells. Importantly, we and others have shown that quiescent cancer cells have a context-dependent oxidative phosphorylation (OXPHOS) dependency that can be pharmacologically targeted (recently reviewed in ${ }^{10}$ ). Previously, we showed that pharmacological inhibition of OXPHOS resulted in selective upregulation of key genes of the initial steps in the cholesterol biosynthesis, namely the mevalonate pathway, in Q-MCTS but not in P-MCTS or monolayer cells. Further, we demonstrated that such vulnerabilities can be exploited to design drug combinations effective only in the specific tissue context, such as synergistic cytotoxicity of nitazoxanide (OXPHOS inhibitor) and simvastatin (mevalonate pathway inhibitor) in quiescent cancer cells. Therefore, differentiating various cellular models, such as P-MCTS, QMCTS, and monolayer cell cultures, might provide insights into targetable context-dependent vulnerabilities in the phenotypically heterogeneous tumor tissue context. The metabolic differences between proliferative and quiescent cancer cells have also been described in other cell systems such as fibroblasts and mammary epithelial cells. ${ }^{11,12}$

Several comparative analyses of monolayer and spheroid cultures addressing their global proteomes have shown differences in adhesion proteins, immune response pathways, matrix metalloproteinase expression pattern, phosphoproteomic pathways, and growth rate. ${ }^{13-15}$ However, the integration of proteome analysis with gene expression data and its functional interpretation in coherence with clinical results remains a challenge.

Here, we present a framework for in-depth proteome analysis of drug-treated 3D cultures. We demonstrate the influence of different culture conditions by profiling the colon cancer cell line HCT116 grown as Q-MCTS, P-MCTS, and monolayer cells. Furthermore, we characterize the effects after treatment with four different OXPHOS inhibitors or simvastatin in the different models. The combination of $3 \mathrm{D}$ cell culture and mass spectrometry-based proteome analysis allowed us to complement previous findings on gene expression profiles and to compare the relative protein changes between the three cellular models.

\section{RESULTS AND DISCUSSION}

The aim of this study was to investigate and compare the proteome of three distinct multicellular colon cancer models 


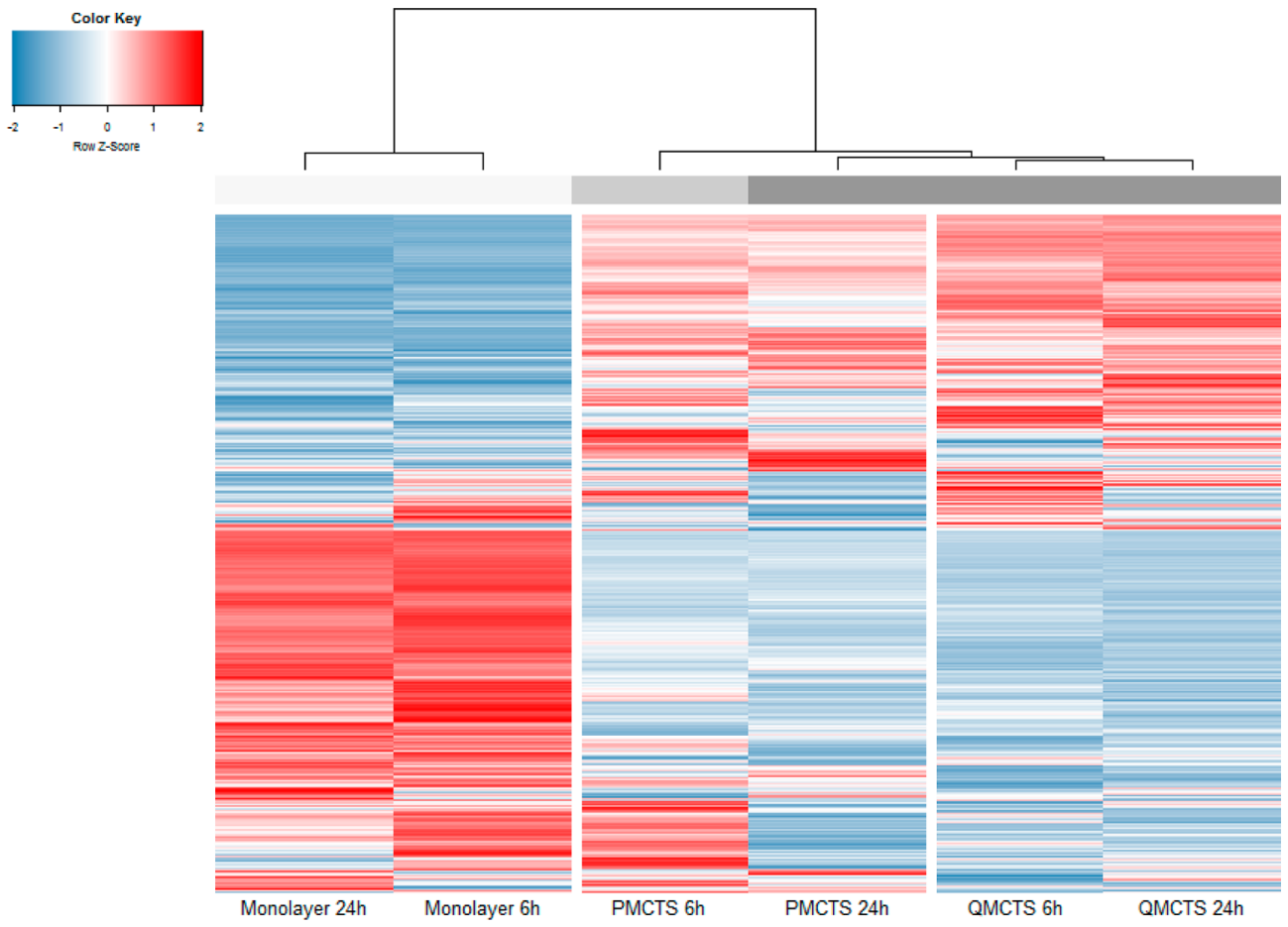

Figure 2. Differences in protein expression between the three cellular models. Comparison of the expression profiles of proteins quantified ( 5965 proteins) in all three cell types (vehicle controls) by hierarchical clustering (1-minus the Pearson correlation, average linkage).

(i.e., P-MCTS, Q-MCTS, and monolayer cell cultures) in response to four different OXPHOS inhibitors (nitazoxanide, FCCP, oligomycin, and salinomycin) or the 3-hydroxyl-3methylglutaryl-coenzym A reductase (HMGCR) inhibitor simvastatin at two different doses. To obtain signatures related to the direct mechanism of action without major feedback signaling, the proteome was studied at a relatively early time point of $6 \mathrm{~h}$ and a later time point of $24 \mathrm{~h}$ for comparison and related to our previous findings on gene expression data with main respect to metabolic pathways. Q-MCTS did not receive fresh media during the culture period, resulting in a smaller fraction of proliferating cells than P-MCTS (Figure S1), which, in turn, translates into differential drug response. ${ }^{8}$

Quality Analysis of Bottom-Up Quantitative Proteomic Data. To obtain quantitative data on protein abundances, proteins from spheroid samples were extracted, digested, and tandem-mass-tag (TMT) labeled using single-pot solid phaseenhanced sample preparation (SP3), as depicted in Figure 7. For in-depth protein profiling, samples were prefractionated by high $\mathrm{pH}$ reverse-phase liquid chromatography prior application to nanoliquid chromatography tandem mass spectrometry (LC-MS/MS). From a total of 268 LC-MS/MS runs, 5174157 MS/MS spectra were obtained yielding 1594878 peptide spectrum matches (PSMS) and 9286 protein groups (Figure 1A). The obtained list of identified proteins was further reduced to proteins with a high-protein false discovery rate (FDR) confidence and at least two unique peptides (Figure 7D). Venn diagram visualization of proteins and protein isoforms present across $60 \%$ of the whole data set revealed that $73 \%$ of identified proteins is shared between monolayer, P-MCTS, and Q-MCTS and 4.3, 6.2, and 1.2\% unique proteins could be found for each cell type, respectively (Figure 1B). When comparing dimethyl sulfoxide (DMSO)treated cells, a similar distribution could be observed (Figure S3B). Comparing proteins expressed by two of the three cell types, monolayer and P-MCTS share 1247 proteins while PMCTS and QMCTS share 828 proteins and QMCTS and monolayer only share 135 proteins. This indicates that monolayer cells and Q-MCTS are most functionally different, corroborating our earlier findings from gene expression analysis. For further data processing, only proteins with unique sequences (no isoforms) were used. The treatment and control data sets with 6025 and 5965 quantified proteins, respectively, were individually processed for downstream analysis. The number of identified and quantified proteins, mass distributions covered, and the reproducibility of the measured absolute protein abundances shown for the control set DMSO data (Pearson correlation coefficient $>0.9$ ) indicate a high identification quality and reproducibility throughout the data set (Figure 1A,C). However, six out of 60 processed samples did not fulfill our expectations with respect to quantification quality (Figure S2) and were removed from further downstream analysis. In Figure 1D, a principal component analysis (PCA) of the whole data set after removal of outliers shows three clusters corresponding to the three different cell types, suggesting that the protein expression of monolayer cells is different to both P-MCTS and Q-MCTS, independently of drug exposure.

Protein Expression Pattern of Three Distinct Multicellular Colon Cancer Models. We recently presented a novel MCTS model that allows to specifically study quiescent cancer cells with highly hypoxic core, low proliferation rate, and low glucose levels resembling dormant tumor regions that present strong differences in gene expression profiles and context-dependent drug responses compared to P-MCTS and monolayer cells. ${ }^{8,9}$ In order to investigate whether these differences are translated to the protein level, we used mass spectrometry (MS)-based proteomics to compare protein expression profiles in the three distinct cellular models. Normalized protein abundances of vehicle (DMSO treated) 


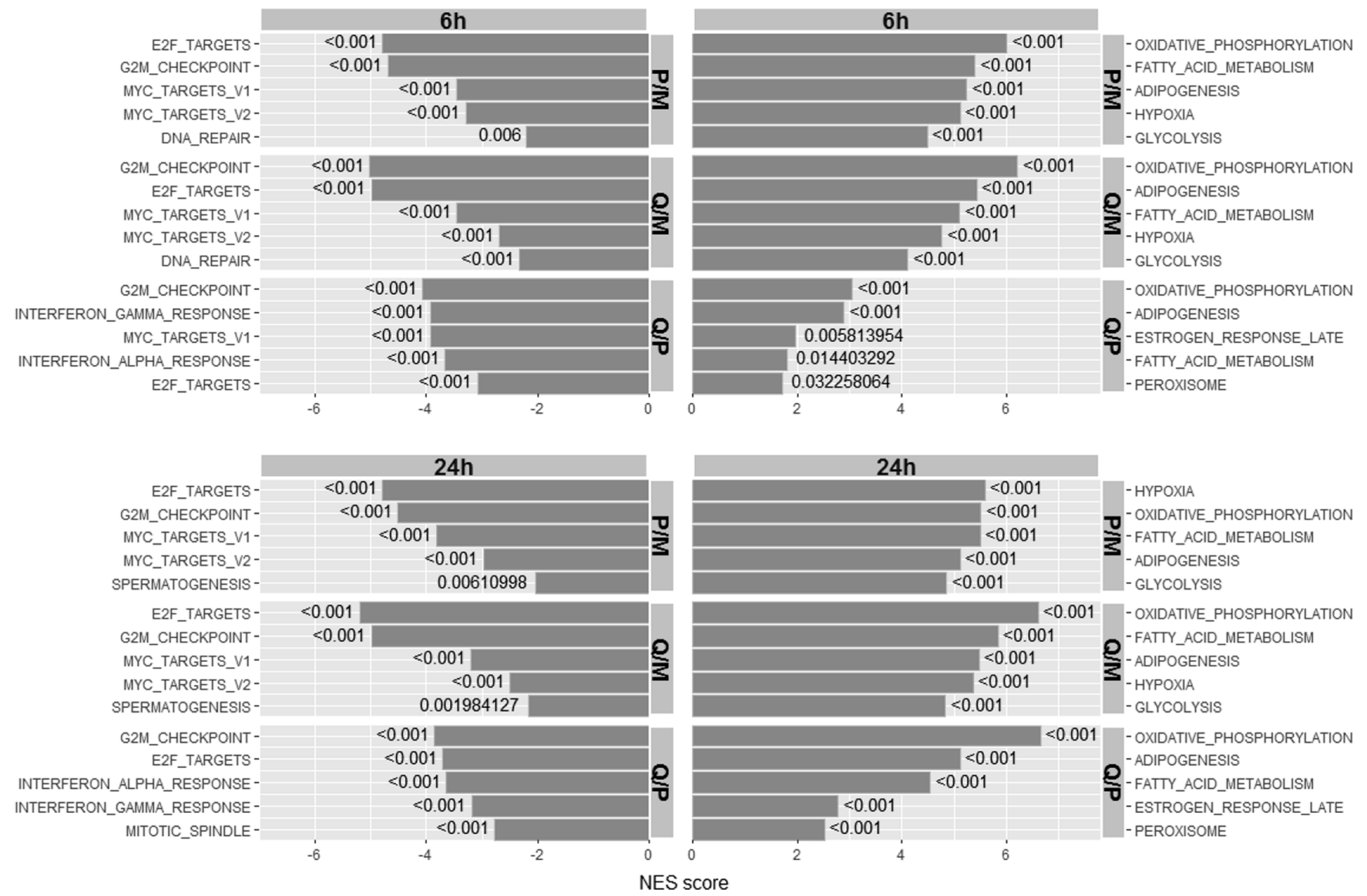

Figure 3. GSEA of the three cellular models. Enrichment analysis of vehicle-treated cells in P-MCTS (P) and Q-MCTS (Q) compared to monolayer (M) cells and Q-MCTS vs P-MCTS comparison. The top five positive and negative enriched pathways are listed for 6 and $24 \mathrm{~h}$ time points. NES scores (normalized enrichment scores) were generated after 1000 permutations. Estimation of the statistical significance of the enrichment score for each respective gene set is indicated by the nominal $p$-value.

controls were hierarchically clustered and represented in a heat map (Figure 2). The cluster analysis shows differences in protein expression between the cellular models, with the largest dissimilarities between monolayer and the spheroid cultures, demonstrating profound differences between $2 \mathrm{D}$ and $3 \mathrm{D}$ cellular models (Figures 2 and S3A). The profiles of respective cellular models clustered together irrespective of the incubation time. However, profiles from P-MCTS differed between the time points whereby profiles of $24 \mathrm{~h}$ incubated $\mathrm{P}$ MCTS clustered with Q-MCTS (6 and $24 \mathrm{~h}$ incubated), indicating that prolonged culture of P-MCTS renders them more similar to Q-MCTS (Figure 2), likely because of depletion of culturing medium and higher levels of cellular quiescence.

To further characterize the differences between the cell models and time effects, we performed gene set enrichment analysis. As expected from the gene expression data and findings by others, ${ }^{8,22}$ P-MCTS and Q-MCTS profiles when compared with monolayer profiles were positively enriched in genes involved in response to hypoxia and OXPHOS and negatively enriched for genes involved in cell cycle progression (E2F targets and G2M checkpoint) (Figure 3). Comparison of Q-MCTS versus P-MCTS revealed that OXPHOS, adipogenesis, fatty acid metabolism, late estrogen response, and peroxisomes were positively enriched, and the pathway G2Mcheckpoint, E2F targets, interferon alpha-and-gamma responses, and mitotic spindle were negatively enriched. This was in line with the results obtained in the gene expression analysis in these cellular models and can be related to OXPHOS as one major source of ATP for quiescent as well as proliferative MCTS. ${ }^{23}$ Overall the enrichment profiles were similar between the different spheroid models and independently of the time point the cells were harvested. In our previous work, the gene expression analysis showed strongest upregulation of the mevalonate pathway genes for Q-MCTS. When comparing the proteome of P-MCTS as well as QMCTS to monolayer cell cultures, cholesterol homeostasis (including the mevalonate pathway proteins) was positively enriched for both time points with slightly higher enrichment scores for P-MCTS (Supporting_Information_XLSX_1). Elevated cholesterol levels have been reported for several solid tumors to play a role in cancer progression and to highly correlate with tumor cell resistance to chemotherapy. ${ }^{24-26}$ However, compared to the strong genetic upregulation in QMCTS, protein-based enrichment analysis demonstrated only weak activation of the mevalonate/cholesterol pathway with more positive enrichment in P-MCTS. Indicating that although the mevalonate pathway genes are overexpressed in both $\mathrm{Q}$ MCTS and P-MCTS, only P-MCTS are metabolically fit to synthesize the proteins. In conclusion, our results highlight the need of metabolically challenged cells to initiate the upregulation of OXPHOS to maintain energy demands and the active downregulation of energy-consuming pathways. Moreover, our results suggest that gene expression does not 
translate to protein expression in metabolically challenged cells.

Protein Expression Pattern of Three Distinct Multicellular Colon Cancer Models Upon Treatment with Four Candidate Compounds Targeting OXPHOS and Simvastatin. In our previous studies, ${ }^{8}$ we showed that QMCTS are more sensitive to OXPHOS inhibition, as they experience low glucose levels and are forced to utilize OXPHOS to maintain their energy needs (contrary to PMCTS and monolayer cells, which persist in high glucose concentrations and can produce enough ATP through glycolysis alone). Based on previous gene expression analysis, we identified the mevalonate pathway as important for the survival of Q-MCTS when inhibiting OXPHOS. Also, we observed potent synergistic toxicity upon combinatory treatment with simvastatin and OXPHOS inhibitors, which highlight the mechanistically coupling of both pathways. ${ }^{8}$ Here, we set out to identify model-specific responses on the protein level upon drug treatment. Therefore, we compared the effects of four different OXPHOS inhibitors: nitazoxanide, FCCP, oligomycin, salinomycin, and simvastatin to DMSO controls at two different drug doses and two different drug exposure times. Gene set enrichment analysis of protein expression after $24 \mathrm{~h}$ drug treatment revealed a negative enrichment of proliferation in monolayer, P-MCTS, and partially in Q-MCTS upon all treatments, whereas an upregulation of hypoxia, metabolic, and immune processes could be observed (Table 1). Furthermore, we observed an evident positive enrichment of cholesterol homeostasis upon treatment with simvastatin and salinomycin in monolayer cells and P-MCTS.

In Figure 4, a detailed analysis of cholesterol homeostasis proteins shows an upregulation of key proteins of the mevalonate pathway such as ACAT2, CYP51A1, HMGCS1, HMGCR, and SQLE upon Simvastatin and Salinomycin treatment in dose response and $24 \mathrm{~h}$ post treatment in monolayer and P-MCTS but not in Q-MCTS. On the protein level, Q-MCTS do not show an enrichment of the mevalonate pathway, as observed in our previous work on the gene expression level (Senkowski et al., 2016, Figure4B).

This indicates that Q-MCTS, maintained in culture medium containing low nutrient concentrations, may not be metabolically viable to synthesize proteins from overexpressed transcripts, and that protein expression $24 \mathrm{~h}$ post treatment indicates only a modest correlation of transcripts and proteins. As protein levels are highly influenced by post-translational events, such as folding, modifications, or degradation processes, which are depending on the energy status of a cell or the cell cycle, they are increasingly affected in metabolically challenged cells. $^{27}$

However, for monolayer cells, the upregulation of key proteins such as HMGCS1, HMGCR, and SQLE upon salinomycin and simvastatin treatment after $24 \mathrm{~h}$ incubation was in line with the gene expression data. This indicates higher susceptibility of monolayer cells to drug treatment and a reduced ability of MCTS to respond to the treatment. MCTS are characterized by complex multicellular interactions that lead to alternated and adapted gene and protein expression in response to nutrient availability. Protein synthesis is one of the most energy-demanding metabolic processes and subject to a strict regulation in starved cells. ${ }^{28,29}$ An overall downregulation of protein synthesis despite selective transcriptional activity upon drug treatment indicates a not fulfilled genetic-intention especially in P-MCTS and Q-MCTS. An upregulation of cholesterol homeostasis proteins upon simvastatin, targeting the rate-limiting enzyme in the mevalonate/cholesterol pathway, (HMGCR) could be explained by a statin-induced feedback upregulation as an intention to maintain stable endproduct levels. $^{24,30}$

Interestingly, positive enrichment of cholesterol homeostasis upon treatment with salinomycin, but not other OXPHOS inhibitors, in monolayer cells and P-MCTS indicates that the underlying molecular mechanism of the upregulation of the cholesterol pathway by salinomycin seems to be different from the other OXPHOS inhibitors.

To identify proteins that are commonly regulated by the individual cell types, we focused on the top five differentially expressed proteins in the three cellular model types after 6 and $24 \mathrm{~h}$ treatment. Frequency analysis comparing different treatments and cell types resulted in the identification of five proteins that were most frequently regulated (Figure 5). We identified dermicidin (P81605), a potential oncogene, which has been associated with tumor growth and increased survival and migration in different tumors, ${ }^{31}$ to be downregulated in all cell types upon drug treatment. The finding of dermicidin has to be interpreted with caution as it had been shown to be present in human skin, sweat, and tears, exhibiting high stability, likely to contaminate throughout the sample workup. ${ }^{32}$ Calcium-binding protein $(45 \mathrm{kDa}$, Q9BRK5) and nucleobindin-1 (Q02818) were downregulated in monolayers, whereas eukaryotic elongation factor 2 kinase (O00418), which has been shown to protect cancer cells against nutrient starvation, $^{33}$ and tripartite motif-containing protein 26 (Q12899) were downregulated specifically in Q-MCTS (Figure 5).

Identification of Early Responding Proteins in QMCTS upon OXPHOS Inhibition. To identify early responding proteins, we performed enrichment analysis using the online tool EnrichR of the most differentially (fold change $>0.6)$ upregulated proteins after treatment with exclusively OXPHOS inhibitors for $6 \mathrm{~h}$. Table 2 shows the 10 most enriched pathways for the three cell types. Proliferative spheroids react with increased oxidative stress upregulating selenocysteine synthesis and termination of eukaryotic translation. We can observe strong context-dependent enrichment in OXPHOS proteins $(p<0.000001)$ exclusively in Q-MCTS. This context-dependent drug response points toward OXPHOS being a vulnerability in quiescent, nutrient-deprived cells. These observations are in line with ATP level measurements in Q-MCTS after $6 \mathrm{~h}$ treatment with nitazoxanide and salinomycin. ATP levels drop to 61 and $71 \%$, for $10 \mu \mathrm{M}$ nitazoxanide or $4 \mu \mathrm{M}$ salinomycin, indicating an early-onset energy crisis (Figure 6). As we found only weak association with OXPHOS protein upregulation for Q-MCTS after $24 \mathrm{~h}$ treatment, this suggests that Q-MCTS after $24 \mathrm{~h}$ are severely depleted of energy and unable to synthesize OXPHOS proteins anymore (Tables 1 and S1).

\section{CONCLUSIONS}

In this study, we combine $3 \mathrm{D}$ spheroid cell culture with a global proteomic approach to investigate cellular responses to inhibitors targeting OXPHOS or the mevalonate pathway. Furthermore, we use SP3 paramagnetic bead technology and isobaric peptide labeling to process multiple samples simultaneously and offline high-pH reversed-phase prefractionation to gain high-quality in-depth proteomic data. With a 


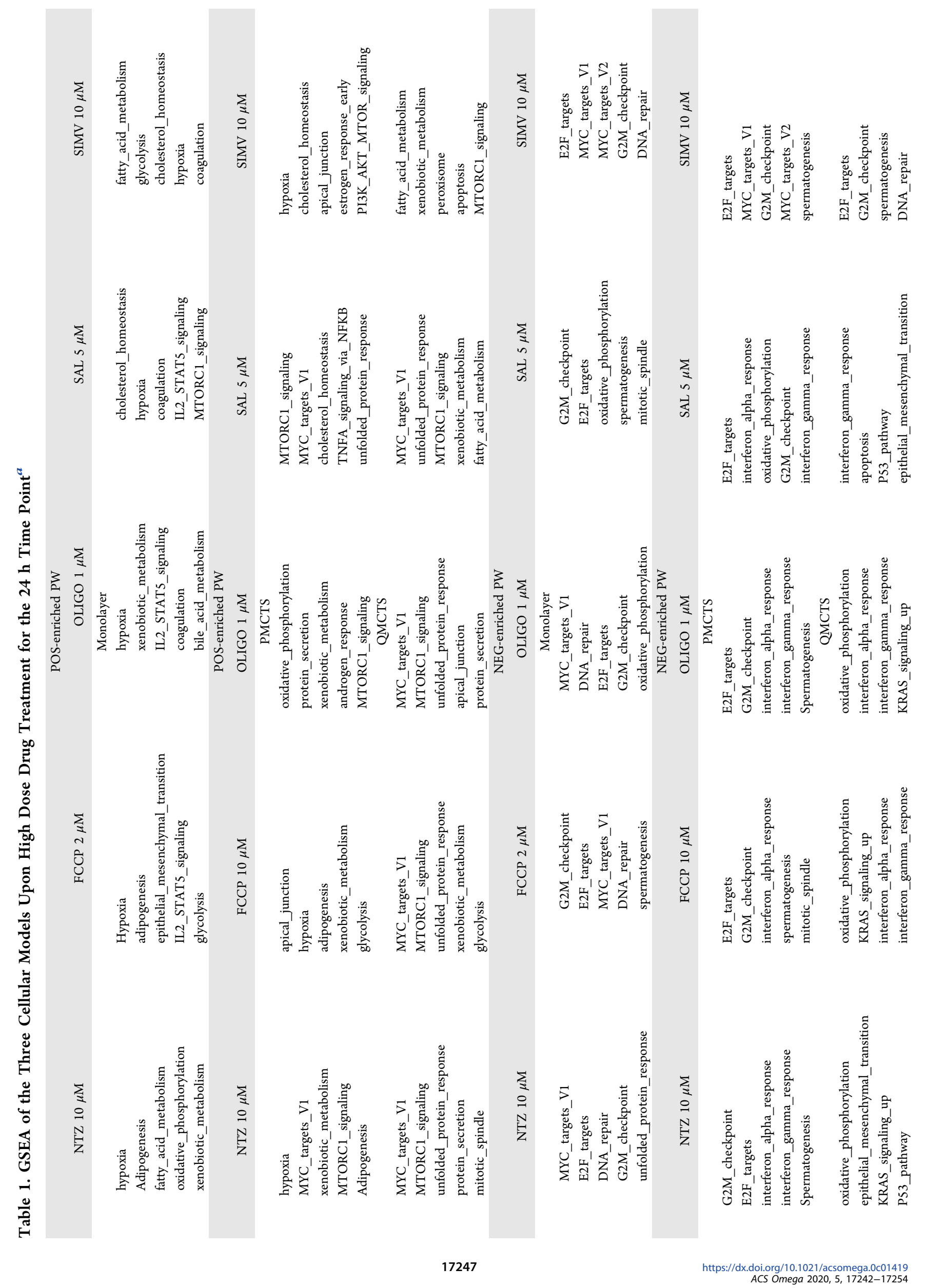


coverage of 9286 protein groups identified, this workflow is suitable for large-scale proteome analysis. ${ }^{34,35}$

Our data on vehicle-treated spheroids confirm the results on the gene level with respect to profound differences in the regulation of hypoxia, OXPHOS, and cell cycle progression between the three cell systems. This underlines the importance of choosing an accurate model system when addressing research questions using cell culture. Moreover, the outlined workflow paves the way for proteomic analysis of other 3D cultures, for example, tumor organoids, derived from primary cancer cells. As organoids enable propagation and expansion of patient-derived cellular material from different tumor types, thus providing enough cellular material for proteomic analysis, our workflow could be applied in these models for studying individualized drug responses on the global proteomic level.

Cancer cell metabolic pathways are a highly complex system consisting of multiple feedback loops that contribute to cancer surveillance. Understanding the vulnerabilities of cancer cells will help to develop successful drugs with increased specificity and efficacy. The present study emphasized the importance of taking the metabolic status and culture conditions into account when performing proteome analysis and drug evaluations. We demonstrated that drug-induced changes in gene expression are not always translated on the protein level, likely because metabolically challenged cells are not able to respond beyond gene expression. The metabolic capacity of the cellular models used is of high importance when searching for drug targets using proteomics under different culture conditions.

Finally, our study shows that proteome analysis is a powerful approach to identify context-dependent drug responses, as exemplified by the demonstrated dependency on OXPHOS specifically seen in Q-MCTS $6 \mathrm{~h}$ post treatment. Insights from this study contribute to a better understanding of the metabolic complexity of proliferative and quiescent cancer cells, and the presented workflow might be applied to future investigations regarding their targetability.

\section{EXPERIMENTAL SECTION}

Materials. Plastic consumables for sample preparation were obtained from Applied Biosystems by Life Technologies (MicroAmp 8-tube Strip, REFN8010580 and MicroAmp 8Cap-strip, REFN8010535). Sera-Mag SpeedBeads used in a 1:1 combination mix for SP3 protein clean-up were obtained from GE Healthcare, UK and prepared and maintained as described elsewhere. ${ }^{16}$ The DynaMag-96 Side magnet was purchased from Life Technologies, Oslo. Reagents for TMT labeling were obtained from Thermo Scientific (TMTsixplex Label Reagent Set, LOT\#QL226165). Restriction enzymes were obtained from Thermo Scientific. Polymeric strong cation-exchange columns were obtained from Phenomenex, Part\# 8B-S029-TAK. Unless specified otherwise, all other reagents were obtained from Sigma Aldrich.

Cell Culture. HCT116 GFP human epithelial colon carcinoma cells, constitutively expressing GFP (Anticancer 2009), were maintained in McCoy's 5A modified medium (Sigma-Aldrich) supplemented with $10 \%$ fetal calf serum, 50 $\mu \mathrm{g} / \mathrm{mL}$ streptomycin, $60 \mu \mathrm{g} / \mathrm{mL}$ penicillin, and $2 \mathrm{mM} \mathrm{L-}$ glutamine at $37{ }^{\circ} \mathrm{C}$ in $5 \% \mathrm{CO}_{2}$.

Monolayer Cultures. HCT116 GFP cells were seeded into 6-well plates (Nunc) at 300000 cells/well and cultivated in McCoy 5A medium for $24 \mathrm{~h}$ before drug treatment.

Spheroid Formation. Spheroids were formed from 5000 HCTS 116 cells in $60 \mu \mathrm{L}$ of fresh medium, plated into 384-well 

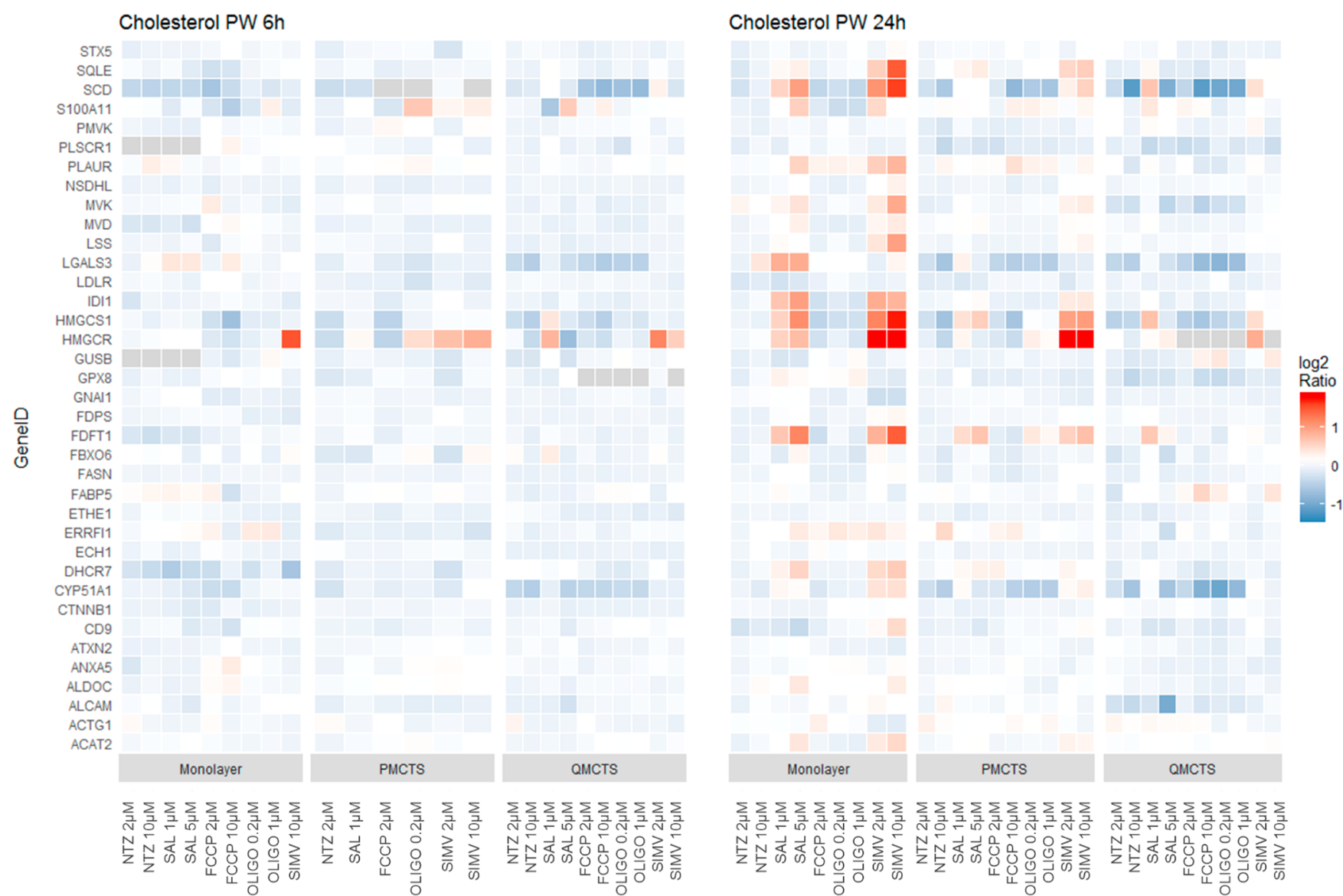

Figure 4. Cholesterol pathway proteins regulated upon drug treatment at 6 and $24 \mathrm{~h}$ time points compared to vehicle control. Protein abundance ratios for respective gene IDs extracted from GSEA hallmark cholesterol homeostasis are depicted.

U-bottom ultralow attachment plates (Corning) using a Biomek 4000 liquid handling system (Beckman Coulter), and subsequently centrifuged at $200 \mathrm{~g}$ for $5 \mathrm{~min}$. Plates were covered with humidified MicroClime Environmental Microplate Lids (Labcyte) and cultured for 7 days at $37{ }^{\circ} \mathrm{C}$ in $5 \%$ $\mathrm{CO}_{2}$. P-MCT culturing involved approximately $97 \%$ medium exchange with fresh medium on day 4 and 7. Q-MCT culturing did not involve any medium changes.

Drug Treatment. Monolayers, $24 \mathrm{~h}$ postseeding, and spheroids, 7 days postseeding, were treated with the OXPHOS inhibitors or simvastatin (at two different concentrations) or DMSO for 6 and $24 \mathrm{~h}$ (Figure 7A), respectively. Each treatment, time point, and concentration were performed as 28 replicates (single spheroids), which were pooled and frozen at $-70{ }^{\circ} \mathrm{C}$ until preparation for MS analysis. Monolayer cells were washed with PBS after 6 or $24 \mathrm{~h}$ treatment, detached using Accutase, pelleted by centrifugation at $200 \mathrm{~g}$ for $5 \mathrm{~min}$, and frozen at $-70{ }^{\circ} \mathrm{C}$.

ATP Measurements in Spheroid Cultures (CellTiter Glo 3D). Compounds were administered to spheroid cultures using Echo liquid handler 550. After $6 \mathrm{~h}$ incubation, the majority of the drug-containing medium was aspirated using ELx405 (BioTek). Then, $50 \mu \mathrm{L}$ of AccuMax (Sigma) was added into each well, and plates were incubated at $37^{\circ} \mathrm{C}$ for 30 min to allow spheroid dissociation. Subsequently, plates were centrifuged for $5 \mathrm{~min}$ at $200 \mathrm{~g}$, and the supernatant was aspirated using ELx405. The plates were then left at room temperature for $30 \mathrm{~min}$ in order to cool down. This was followed by the addition of $25 \mu \mathrm{L}$ CellTiter-Glo 3D solution
(Promega) into each well. Plates were then incubated at room temperature on an orbital shaker set to $900 \mathrm{rpm}$ for $10 \mathrm{~min}$ to allow even disruption of cell pellets. Subsequently, plates were incubated at room temperature for $20 \mathrm{~min}$, and luminescence was measured using automated plate reader FLUOstar OMEGA (BMG Labtech).

Immunological Staining. Spheroids were washed with PBS, fixed with $4 \%$ formalin in PBS, embedded in paraffin, and sectioned. Stainings for Ki67 performed using the Autostainer 480 (Thermo Fisher Scientific). Mouse anti-Ki67 clone MIB1 (\#M7240) antibody was purchased from Dako AB. Slides were first incubated with Ultra V block (TA-125-UB, Thermo Fisher Scientific) for $5 \mathrm{~min}$ and thereafter incubated with primary antibodies at 1:1000 dilution for $30 \mathrm{~min}$. The slides were then incubated with labeled horseradish peroxidase polymer for 30 min, followed by 3,3'-diaminobenzi-dine (DAB Quanto) solution for $5 \mathrm{~min}$. Slides were counterstained in Mayer's hematoxylin (01820; Histolab) for 5 min using Autostainer XL (Leica) and then rinsed in lithium carbonate water (diluted 1:5 from saturated solution) for $1 \mathrm{~min}$. The slides were dehydrated in graded ethanol and coverslipped (PERTEX; Histolab) using an automated glass coverslipper (CV5030; Leica). The slides were scanned using the automated scanning system Aperio AT2 (Aperio Technologies).

Sample Preparation for MS Analysis. Cell Lysis, Digestion, and TMT Labeling. Cell pellets were resuspended in $200 \mu \mathrm{L}$ of lysis buffer [50 mM triethylammonium bicarbonate (TEAB), $1 \%$ sodium dodecyl sulfate, and protease inhibitor] and subsequently incubated at $95{ }^{\circ} \mathrm{C}$ for $5 \mathrm{~min}$. 

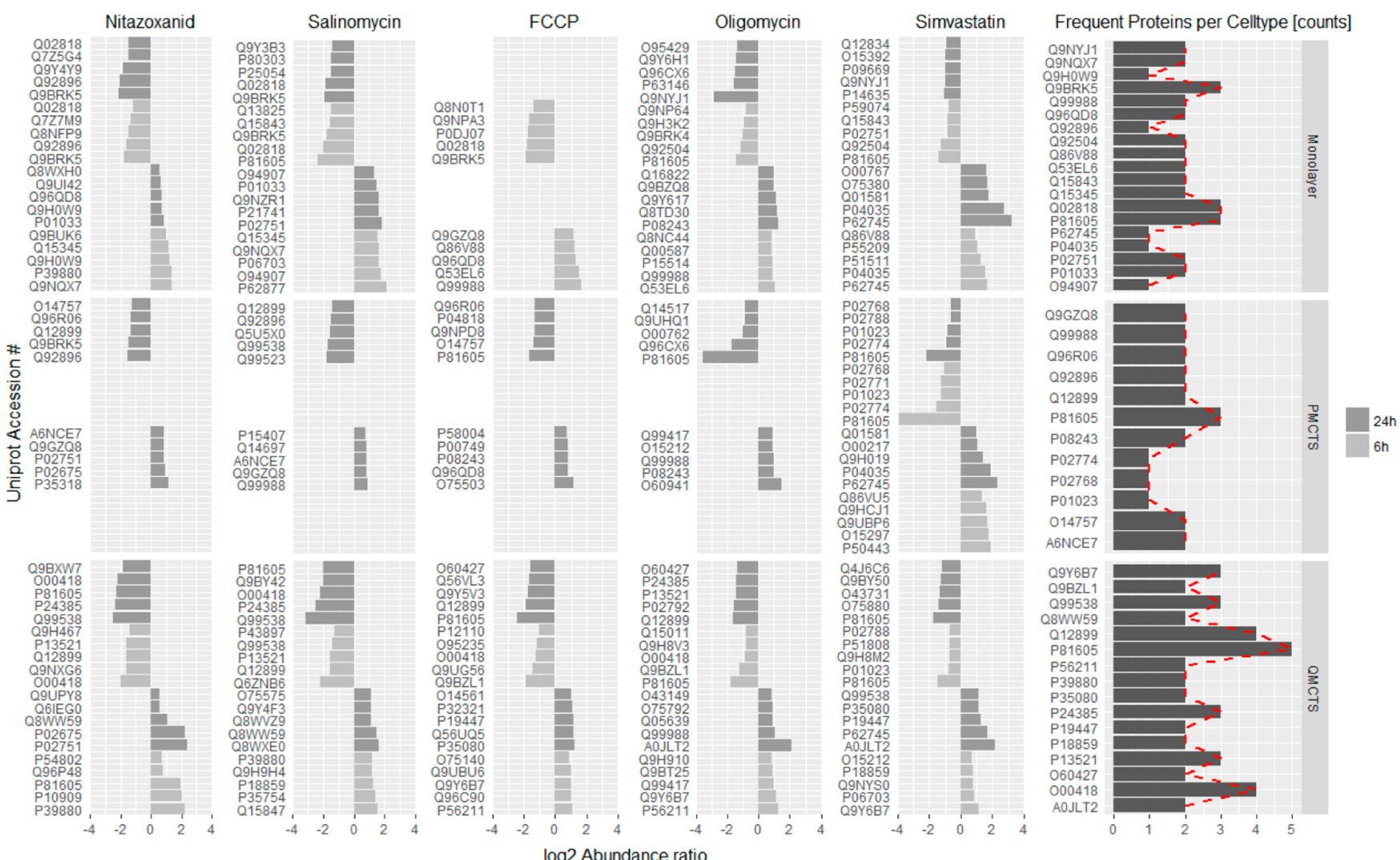

Figure 5. Top five differentially expressed proteins upon 6 and $24 \mathrm{~h}$ high dose drug treatment. Proteins regulated upon any treatment and appearing at least two times in each cell type are listed in the right part of the figure. The dark gray bars represent the frequency of how often a protein was differentially regulated upon treatment for the respective cell type (a count of five indicates a protein being regulated upon all five treatments). Frequency analysis focusing on proteins with counts three and higher revealed five proteins of interest that show cell type-specific regulations: P81605 (regulated upon all treatments and in all cell types), Q9BRK5, Q02818 (regulated upon nitazoxanid, FCCP, and salinomycin in monolayers), O00418, and Q12899 (regulated upon OXPHOS inhibitors in Q-MCTS).

Table 2. Pathways Upregulated Upon OXPHOS Inhibition after $6 \mathrm{~h}$ Treatment ${ }^{a}$

\begin{tabular}{|c|c|c|c|c|c|}
\hline \multicolumn{2}{|l|}{ monolayer } & \multicolumn{2}{|l|}{ PMCTS } & \multicolumn{2}{|l|}{ QMCTS } \\
\hline pathway & $p$-value & pathway & $p$-value & pathway & $p$-value \\
\hline $\begin{array}{l}\text { cytosolic sulfonation } \\
\text { of small molecules }\end{array}$ & 0.0012 & $\begin{array}{l}\text { intra-Golgi and retrograde Golgi-to- } \\
\text { ER traffic }\end{array}$ & 0.0022 & $\begin{array}{l}\text { respiratory electron transport, ATP synthesis by } \\
\text { chemiosmotic coupling, and heat production by } \\
\text { uncoupling proteins }\end{array}$ & $6.91 \times 10^{-6}$ \\
\hline phase II conjugation & 0.0029 & $\begin{array}{l}\text { retrograde transport at the trans- } \\
\text { Golgi network }\end{array}$ & 0.0023 & the citric acid cycle and respiratory electron transport & $3.57 \times 10^{-5}$ \\
\hline platelet degranulation & 0.0033 & membrane trafficking & 0.0030 & respiratory electron transport & $6.37 \times 10^{-5}$ \\
\hline $\begin{array}{l}\text { response to elevated } \\
\text { platelet cytosolic } \\
\mathrm{Ca} 2\end{array}$ & 0.0038 & vesicle-mediated transport & 0.0053 & complex I biogenesis & $2.35 \times 10^{-4}$ \\
\hline complex I biogenesis & 0.0086 & peptide chain elongation & 0.0066 & RHO GTPases activate CIT & $6.90 \times 10^{-4}$ \\
\hline $\begin{array}{l}\text { apoptotic execution } \\
\text { phase }\end{array}$ & 0.0093 & viral mRNA translation & 0.0066 & prefoldin-mediated transfer of substrate to $\mathrm{CCT} / \mathrm{TriC}$ & $1.98 \times 10^{-3}$ \\
\hline apoptosis & 0.0113 & selenocysteine synthesis & 0.0070 & signaling by Rho GTPases & $1.99 \times 10^{-3}$ \\
\hline $\begin{array}{l}\text { programmed cell } \\
\text { death }\end{array}$ & 0.0118 & eukaryotic translation termination & 0.0070 & $\begin{array}{l}\text { cooperation of prefoldin and TriC/CCT in actin and tubulin } \\
\text { folding }\end{array}$ & $2.78 \times 10^{-3}$ \\
\hline signaling by MST1 & 0.0142 & eukaryotic translation elongation & 0.0074 & RHO GTPase effectors & $3.49 \times 10^{-3}$ \\
\hline $\begin{array}{l}\text { amyloid fiber } \\
\text { formation }\end{array}$ & 0.0161 & $\begin{array}{l}\text { nonsense-mediated decay } \\
\text { independent of the exon junction } \\
\text { complex }\end{array}$ & 0.0074 & HIV life cycle & $4.99 \times 10^{-3}$ \\
\hline
\end{tabular}

${ }^{a}$ Proteins that were upregulated with fold change $>0.6$ upon OXPHOS inhibition were analyzed using EnrichR.

Samples were tip-sonicated at high frequency on ice with a tipsonifier (Bandelin, Sonopuls UW 2070, Berlin) for $1.5 \mathrm{~min}$ in three $30 \mathrm{~s}$ increments until complete cell lysis was achieved. Samples were centrifuged in an Eppendorf benchtop centrifuge at $4{ }^{\circ} \mathrm{C}, 14,000 \mathrm{~g}$ for $20 \mathrm{~min}$. Protein concentration of the supernatants was measured by the bicinchoninic acid (Thermo Fisher) assay according to manufacturer's instructions. Complete supernatants were reduced and alkylated with 10 $\mathrm{mM}$ dithiothreitol (DTT) at $45{ }^{\circ} \mathrm{C}$ for $30 \mathrm{~min}$ and $40 \mathrm{mM}$ iodoacetamide at room temperature for $30 \mathrm{~min}$ in the dark. 

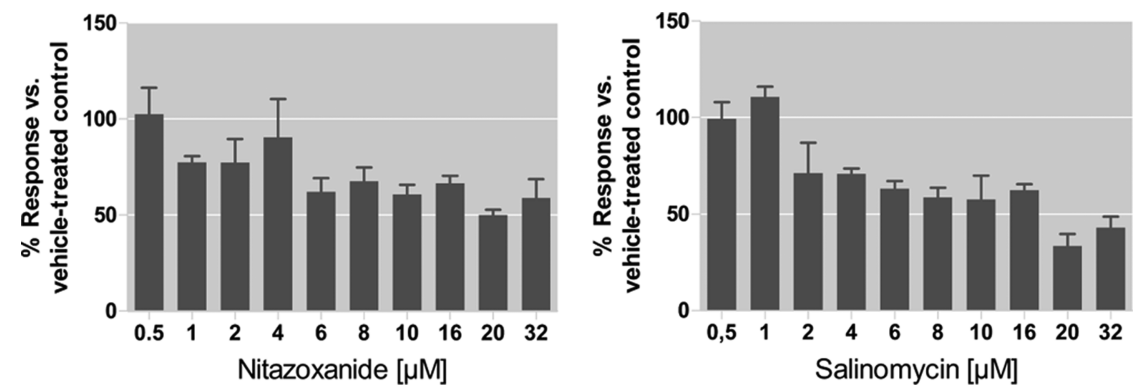

Figure 6. ATP measurements in Q-MCTS after $6 \mathrm{~h}$ treatment with nitazoxanide and salinomycin. ATP levels (mean \pm SD of technical triplicates) are presented as percentage (\%) of vehicle-treated control (100\%). This graph was prepared using GraphPad Prism v5.

\begin{tabular}{ccccc}
\hline Drug & $\begin{array}{c}\text { Dose } \\
{[\mu \mathrm{MM}]}\end{array}$ & Details & Formula & Pathway \\
\hline FCCP & 2,10 & Mitochondrial effects & $\mathrm{C}_{10} \mathrm{H}_{5} \mathrm{~F}_{3} \mathrm{~N}_{4} \mathrm{O}$ & OXPHOS \\
$\begin{array}{c}\text { Nitazoxanide } \\
\text { (NTZ) }\end{array}$ & 2,10 & $\begin{array}{c}\text { Anthelminthic, antiviral, } \\
\text { antiprotozoal }\end{array}$ & $\mathrm{C}_{12} \mathrm{H}_{6} \mathrm{~N}_{3} \mathrm{O}_{5} \mathrm{~S}$ & OXPHOS \\
$\begin{array}{c}\text { Oligonycin } \mathrm{A} \\
\text { (OLIGO) }\end{array}$ & $0.2,1$ & $\begin{array}{c}\text { Mitochondrial effects } \\
\text { A }\end{array}$ & $\mathrm{C}_{45} \mathrm{H}_{74} \mathrm{O}_{11}$ & OXPHOS \\
$\begin{array}{c}\text { Salinomycin } \\
\text { (SAL) }\end{array}$ & 1,5 & Antibacterial & $\mathrm{C}_{42} \mathrm{H}_{70} \mathrm{O}_{11}$ & OXPHOS \\
$\begin{array}{c}\text { Simvastatin } \\
\text { (SIMV) }\end{array}$ & 2,10 & $\begin{array}{c}\text { HMG-COA reductase } \\
\text { inhibitor }\end{array}$ & $\mathrm{C}_{25} \mathrm{H}_{38} \mathrm{O}_{5}$ & MEVALONATE \\
\hline
\end{tabular}

C

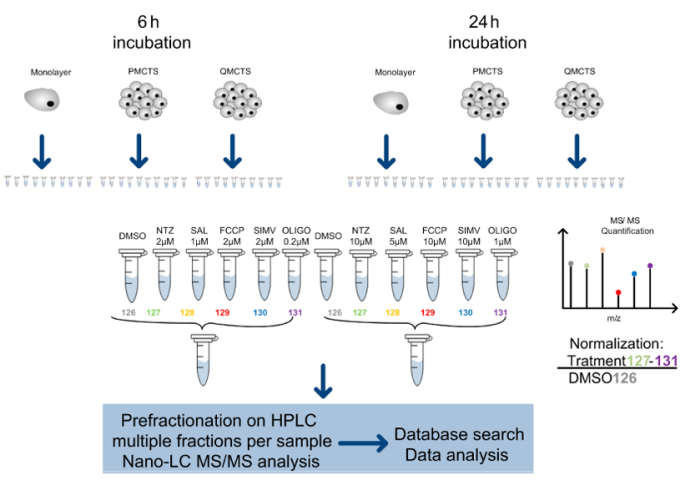

B
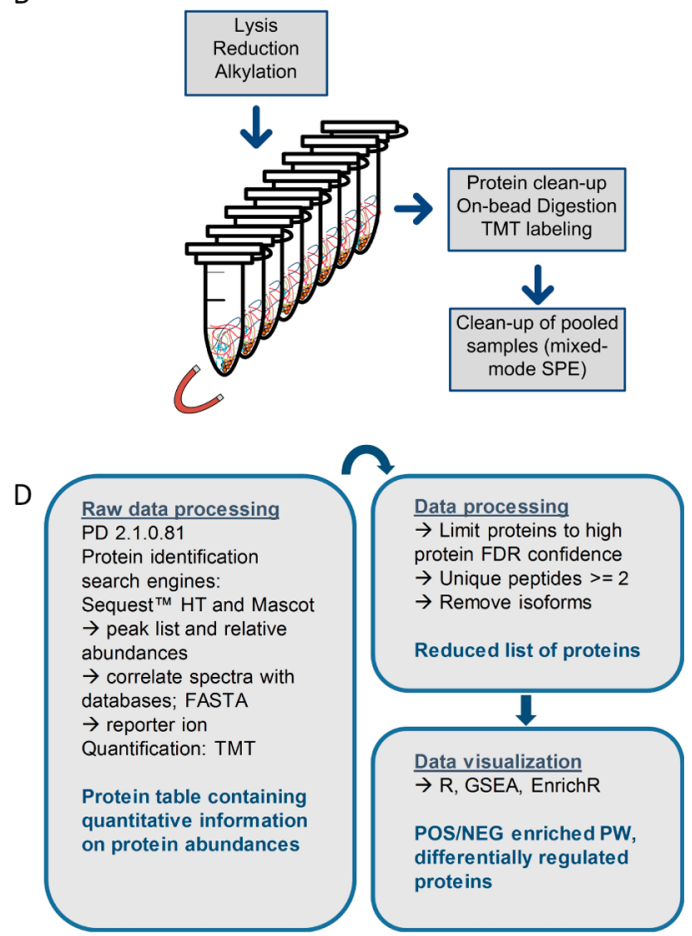

Figure 7. Workflow and experimental design of nano-LC-MS/MS-based proteomics for 2D and 3D colon cancer cells. HCT116 cells were cultured as 2D monoculture or 3D spheroids in 384 well plates. Cells were treated with indicated concentrations of the respective drugs (A) or vehicle control for 6 or $24 \mathrm{~h}$. For proteome analysis, cells of 28 spheroid replicates were pooled, and proteins were extracted, digested, TMTlabeled, and purified by SP3 paramagnetic bead technology, followed by high-pH reversed-phase prefractionation prior nano-LC-MS/MS analysis $(B, C)$. Acquired peptide spectra data were processed using proteome discoverer software, and quantified proteins were visualized using $\mathrm{R}$ and open-source platforms like GSEA and EnrichR (D). This graphic was generated in Microsoft Visio 2007.

Following this, the alkylation reaction was quenched by a second reduction step resulting in a final DTT concentration of $20 \mathrm{mM}$ for $20 \mathrm{~min}$ at $37{ }^{\circ} \mathrm{C}$.

For TMT labeling and sample clean-up, paramagnetic beads were used, and sample processing was based on the method described by Hughes et al., 2014, ${ }^{16}$ as illustrated in Figure 7B. Briefly, samples were transferred to PCR strips, and $30 \mu \mathrm{g}$ of protein was evaporated to dryness in a SpeedVac evaporator (SCANVAC, labogene). Proteins were resuspended in $8 \mu \mathrm{L}$ of $50 \mathrm{mM}$ TEAB buffer supplemented with $2 \mu \mathrm{L}$ of magnetic bead mix and $20 \mu \mathrm{L}$ of acetonitrile (ACN)/formic acid (FA) solution to obtain a final concentration of $66 \% \mathrm{ACN}$ and $0.25 \%$ FA. After $8 \mathrm{~min}$ incubation at room temperature, followed by 2 min incubation on a magnetic rack, supernatants were removed. The bead-bound proteins were washed twice with $70 \%$ ethanol, once with ACN, and subsequently air-dried for $30 \mathrm{~s}$. On-bead digestion was carried out with trypsin and
Lys-C enzymes in 1:25 ratio in $25 \mu \mathrm{L}$ of $50 \mathrm{mM}$ TEAB buffer overnight at $37^{\circ} \mathrm{C}$. Supernatants were collected, and peptides were labeled with the TMT 6-plex reagent for $3 \mathrm{~h}$ at room temperature according to manufacturer's specification. Digestion and labeling efficiency was tested on 16 randomly selected samples whereby approximately $95 \%$ zero-missed cleavages and $99 \%$ labeling efficiency were found. The labeling scheme is depicted in Figure $7 \mathrm{C}$. The labeling reaction was quenched with Tris-HCL-buffer, $\mathrm{pH} 8$ for $30 \mathrm{~min}$ at $37^{\circ} \mathrm{C}$, and samples were pooled resulting in 12 6-plex pools (five treatments in two concentrations and one DMSO control, three cell types, and two time points) for the treatment experiment. In order to compare baseline proteomes of the three cell types, one additional 6-plex pool containing vehicle-control samples of each cell type and time point was prepared, which will be further referred to as the control set. ACN excess was evaporated, and remaining reagents and detergents were 
removed by mixed-mode (reversed-phase cation exchange) solid-phase extraction (StrataXC, 8B-S029-TAK-TN). Samples were evaporated and stored at $-20{ }^{\circ} \mathrm{C}$ until high-pH reversedphase prefractionation.

Peptide Prefractionation by High-pH Reversed-Phase Liquid Chromatography. Peptide prefractionation was performed for labeled samples by high-pH reversed-phase liquid chromatography using an Agilent 1200 HPLC system. Samples were reconstituted in $80 \mu \mathrm{L}$ of mobile phase A (20 $\mathrm{mM}$ HPLC-grade ammonia) and loaded on an XBridge BEH300 C18, $3.5 \mu \mathrm{m}, 1 \times 150 \mathrm{~mm}$ column (Waters, part number: 186003606$)$. Over a $70 \mathrm{~min}$ gradient with gradually increasing mobile phase B (10 mM ammonia, $80 \% \mathrm{ACN})$ up to $100 \%$ at a flow rate of $0.12 \mathrm{~mL} / \mathrm{min}, 96$ fractions per sample were collected and subpooled into 21 fractions per sample and subsequently dried.

Nano LC-MS/MS Analysis. The labeled peptides dissolved in 20 and $4 \mu \mathrm{L}$ were injected and separated using an Ultimate 3000 RSLCnano system (Dionex Ultimate-3000, Thermo Scientific, Sunnyvale CA, USA) set up in a trap and elute configuration. The peptides were trapped on a precolumn (75 $\mu \mathrm{m} \times 20 \mathrm{~mm} 3 \mu \mathrm{m} 100 \AA ̊$ C18 particles, Acclaim PepMap 100, Thermo Scientific) before eluting the peptides onto a $50 \mathrm{~cm}$ EasySpray $50 \mathrm{~cm}$ PepMap RSLC C-18 column (ES803, Thermo Scientific, Bremen, Germany) using LC gradient buffers consisting of A ( $3 \% \mathrm{ACN}, 0.1 \% \mathrm{FA}$ in LC-MS grade water) and $\mathrm{B}$ ( $96 \% \mathrm{ACN}, 0.1 \% \mathrm{FA}$ in LC-MS grade water). The buffer B gradually increased from 3 to $40 \%$ in $98 \mathrm{~min}$ and after that to $99 \%$ in the next $14 \mathrm{~min}$. The column was equilibrated for an additional $11 \mathrm{~min}$ before the next sample was injected. Peptides were eluted at a flow rate of $250 \mathrm{~nL} / \mathrm{min}$ and equilibrated at $350 \mathrm{~nL} / \mathrm{min}$. LC-MS data were acquired using a high-resolution quadrupole orbitrap mass spectrometer (Q-Exactive, Thermo Scientific, Bremen, Germany) in a datadependent mode, where the top 10 most intense precursor ions were selected and fragmented using a normalized collision energy of 30. Full MS spectra were acquired at 70k resolution and fragmentation (MS/MS) spectra at $17.5 \mathrm{k}$ resolution.

Data Analysis. Raw Data Processing. Proteome Discoverer software (Thermo Fisher Scientific, version 2.1.0.81) was used for data analysis. UniProt human database (2015-0924 uniprot-all_Human 42136_Can_Iso.fasta) was used in database searching. The RAW $\overline{\text { data }}$ file MS/MS spectra were filtered for the top 12 peaks per $100 \mathrm{Da}$ mass windows and then subjected to SequestHT search and Percolator validation. Trypsin with full cleavage specificity and two maximum missed cleavage sites was specified for the search. Precursor and fragment mass tolerances were $15 \mathrm{ppm}$ and $0.03 \mathrm{Da}$, respectively. Carbamidomethylation (C) and TMT 6plex (any $\mathrm{N}$ terminus, $\mathrm{K}$ ) were set as static modifications. Methionine oxidation, protein $\mathrm{N}$-terminal acetylation, and deamidation (NQ) were set as dynamic modifications. The database search protein identification acceptance criteria were, peptide-level FDR less than $1 \%$ based on Percolator scoring, a minimal number of peptide sequence per protein of 1 (rank 1 peptide only), and peptides were counted only in top scored proteins. The protein grouping was enabled, and the strict maximum parsimony principle was applied. For reporter ionbased quantification, Quan value corrections were enabled, and the coisolation threshold and average reporter $\mathrm{S} / \mathrm{N}$ threshold were set to 50 and 10 , respectively. The confidence threshold for protein FDR validation was set to $1 \%$, total peptide amount was used for normalization, and obtained normalized abundances were used to determine alterations in expression based on TMT ratios: treatment/DMSO control (127-131)/ 126 or one entire control set, where the DMSO control of all cell types and time points were compared (127-131)/126.

Data Filtering. The data were loaded into the statistical software environment $\mathrm{R}$ v1.0.153. The protein list was filtered for high-protein FDR confidence, and a minimum of two unique peptides per identified protein was allowed, and isoforms and invalid values were removed. Finally, contaminants were removed using a contaminant list obtained from (http://lotus1.gwdg.de/mpg/mmbc/maxquant_input.nsf/ 7994124a4298328fc125748d0048fee2/\$FILE/contaminants. fasta). For more information, see the Results and Discussion section, and protein lists are found in the Supporting Information.

Gene Set Enrichment Analysis. Normalized abundances of protein-coding genes were extracted for each DMSO-treated control, and drug-treated sample ratios were calculated, and rows containing invalid values were removed. Gene set enrichment analysis was performed using the default settings for pre-ranked enrichment analysis (GSEA 3.0) using Hallmarks (h.all.v6.1.symbols.gmt) as the database, NES scores were generated after 1000 permutations, statistics $=$ classic. $^{17,18}$ For the identification of enriched pathways upon exclusively OXPHOS inhibitor treatment at the early $6 \mathrm{~h}$ time point, simvastatin treatment was removed from the data set, and differentially upregulated proteins with a fold change $>0.6$ were inserted to the online tool EnrichR. ${ }^{19,20}$

Data and Software Availability. The MS proteomic data have been deposited to the ProteomeXchange Consortium via the PRIDE ${ }^{21}$ partner repository (http://www.ebi.ac.uk/pride/ archive/) with the data set identifier PXD016495. Username: reviewer43746@ebi.ac.uk, Password: qRsMXAAd.

\section{ASSOCIATED CONTENT}

\section{Supporting Information}

The Supporting Information is available free of charge at https://pubs.acs.org/doi/10.1021/acsomega.0c01419.

Immunohistochemical staining of Ki-67, data quality analysis, differences between the three cell types upon DMSO treatment, and pathways upregulated upon OXPHOS inhibition after $24 \mathrm{~h}$ treatment (PDF)

GSEA CTRL data set (XLSX)

Whole proteome obtained after PD database search (XLSX)

Proteins filtered for a high-protein FDR confidence, at least two unique peptides and unique sequences. The table contains normalized abundances for each sample and TMT ratios for each treatment to respective DMSO control. Protein accession $=6025$ (XLSX)

Proteins filtered for a high-protein FDR confidence, at least two unique peptides and unique sequences. The table contains normalized abundances for the control set (DMSO controls for 6 and $24 \mathrm{~h}$ of all three cell types) after removal of any invalid values. Protein accession $=$ 5965 (XLSX)

Proteins filtered for a high-protein FDR confidence, at least two unique peptides. The table contains TMT ratios for each treatment to the respective DMSO control. Protein accession, including isoforms $=16,938$ (XLSX) 


\section{AUTHOR INFORMATION}

\section{Corresponding Author}

Kim Kultima - Department of Medical Sciences, Clinical Chemistry, Uppsala University, Uppsala SE-751 85, Sweden; Email: kim.kultima@medsci.uu.se

\section{Authors \\ Julia Steinmetz - Division of Rheumatology, Department of Medicine, Solna, Karolinska Institutet and Karolinska University Hospital, SE-171 76 Stockholm, Sweden; () orcid.org/0000-0001-6041-6401 \\ Wojciech Senkowski - Department of Medical Sciences, Cancer Pharmacology and Computational Medicine, Uppsala University, Uppsala SE-751 05, Sweden \\ Johan Lengqvist - Department of Oncology-Pathology, Karolinska Institutet, Stockholm SE-171 77, Sweden \\ Jenny Rubin - Department of Medical Sciences, Cancer Pharmacology and Computational Medicine, Uppsala University, Uppsala SE-751 05, Sweden \\ Elena Ossipova - Division of Rheumatology, Department of Medicine, Solna, Karolinska Institutet and Karolinska University Hospital, SE-171 76 Stockholm, Sweden \\ Stephanie Herman - Department of Medical Sciences, Clinical Chemistry, Uppsala University, Uppsala SE-751 85, Sweden \\ Rolf Larsson - Department of Medical Sciences, Cancer Pharmacology and Computational Medicine, Uppsala University, Uppsala SE-751 05, Sweden \\ Per-Johan Jakobsson - Division of Rheumatology, Department of Medicine, Solna, Karolinska Institutet and Karolinska University Hospital, SE-171 76 Stockholm, Sweden \\ Mårten Fryknäs - Department of Medical Sciences, Cancer Pharmacology and Computational Medicine, Uppsala University, Uppsala SE-751 05, Sweden}

Complete contact information is available at: https://pubs.acs.org/10.1021/acsomega.0c01419

\section{Author Contributions}

J.L., K.K., W.S., M.F., and J.S. contributed to study conception and design. W.S. cultured monolayer and spheroid cells and performed the treatment experiment. W.S. performed the ATP measurements. J.S., J.L., and J.R. performed the sample preparation. J.S., J.L., K.K., and E.O. performed HPLC prefractionation and nano-LC-MS/MS analysis. J.L. and J.S. performed the PD-based database search. J.S. and S.H. analyzed and visualized the data. J.S., W.S., K.K., and M.F. drafted the manuscript. The manuscript was critically revised through contributions of J.S., W.S., K.K., M.F., S.H., J.L., J.R., E.O., P.J.J., and R.L. All the authors have given approval to the final version of the manuscript.

\section{Funding}

(R.L., M.F., and W.S.) This study was supported by the Swedish Cancer Society, Swedish Research Council, the Lions Cancer Research Fund, (J.S and P.J.J.) the Else KrönerFresenius-Stiftung (Else Kröner-Fresenius-Graduiertenkolleg), the Swedish Cancer Society (grant no. CAN 2016/739), and the Swedish Research Council (grant nos. 2015-03955, 201701391, and 2017-02577).

\section{Notes}

The authors declare no competing financial interest.

\section{ABBREVIATIONS}

OXPHOS, oxidative phosphorylation
P-MCTS, proliferative multicellular tumor spheroids Q-MCTS, quiescent multicellular tumor spheroids SP3, single-pot solid phase-enhanced sample preparation TMT, tandem mass tag GFP, green fluorescence protein DMSO, dimethyl sulfoxide

DTT, dithiothreitol

TEAB, triethylammonium bicarbonate HPLC, high $\mathrm{pH}$ liquid chromatography $\mathrm{FDR}$, false discovery rate LC-MS/MS, liquid chromatography tandem mass spectrometry

GSEA, gene set enrichment analysis

HMGCR, 3-hydroxyl-3-methylglutaryl-coenzym A reductase HMGCS, hydroxymethylglutaryl-coenzym A-synthase

MS, mass spectrometry

PCA, principal component analysis

ACN, acetonitrile

FA, formic acid

\section{REFERENCES}

(1) Oudin, M. J.; Weaver, V. M. Physical and Chemical Gradients in the Tumor Microenvironment Regulate Tumor Cell Invasion, Migration, and Metastasis. Cold Spring Harbor Symp. Quant. Biol. 2016, 81, 189-205.

(2) Riffle, S.; Hegde, R. S. Modeling tumor cell adaptations to hypoxia in multicellular tumor spheroids. J. Exp. Clin. Cancer Res. 2017, 36, 102.

(3) Chen, W.; Dong, J.; Haiech, J.; Kilhoffer, M. C.; Zeniou, M. Cancer Stem Cell Quiescence and Plasticity as Major Challenges in Cancer Therapy. Stem Cells Int. 2016, 2016, 1740936.

(4) Hamilton, G.; Rath, B. Applicability of tumor spheroids for in vitro chemosensitivity assays. Expert Opin. Drug Metab. Toxicol. 2019, $15,15-23$.

(5) Brancato, V.; Oliveira, J. M.; Correlo, V. M.; Reis, R. L.; Kundu, S. C. Could 3D models of cancer enhance drug screening? Biomaterials 2020, 232, 119744.

(6) Tuveson, D.; Clevers, H. Cancer modeling meets human organoid technology. Science 2019, 364, 952-955.

(7) Hirschhaeuser, F.; Menne, H.; Dittfeld, C.; West, J.; MuellerKlieser, W.; Kunz-Schughart, L. A. Multicellular tumor spheroids: an underestimated tool is catching up again. J. Biotechnol. 2010, 148, 315

(8) Senkowski, W.; Jarvius, M.; Rubin, J.; Lengqvist, J.; Gustafsson, M. G.; Nygren, P.; Kultima, K.; Larsson, R.; Fryknäs, M. Large-scale gene expression profiling platform for identification of contextdependent drug responses in multicellular tumor spheroids. Cell Chem. Biol. 2016, 23, 1428-1438.

(9) Senkowski, W.; Zhang, X.; Olofsson, M. H.; Isacson, R.; Hoglund, U.; Gustafsson, M.; Nygren, P.; Linder, S.; Larsson, R.; Fryknas, M. Three-dimensional cell culture-based screening identifies the anthelmintic drug nitazoxanide as a candidate for treatment of colorectal cancer. Mol. Cancer Ther. 2015, 14, 1504-1516.

(10) Sica, V.; Bravo-San Pedro, J. M.; Stoll, G.; Kroemer, G. Oxidative phosphorylation as a potential therapeutic target for cancer therapy. Int. J. Cancer 2020, 146, 10-17.

(11) Lemons, J. M. S.; Feng, X.-J.; Bennett, B. D.; Legesse-Miller, A.; Johnson, E. L.; Raitman, I.; Pollina, E. A.; Rabitz, H. A.; Rabinowitz, J. D.; Coller, H. A. Quiescent fibroblasts exhibit high metabolic activity. PLoS Biol. 2010, 8, No. e1000514.

(12) Coloff, J. L.; Murphy, J. P.; Braun, C. R.; Harris, I. S.; Shelton, L. M.; Kami, K.; Gygi, S. P.; Selfors, L. M.; Brugge, J. S. Differential Glutamate Metabolism in Proliferating and Quiescent Mammary Epithelial Cells. Cell Metab. 2016, 23, 867-880.

(13) He, W.; Kuang, Y.; Xing, X.; Simpson, R. J.; Huang, H.; Yang, T.; Chen, J.; Yang, L.; Liu, E.; He, W.; Gu, J. Proteomic comparison of $3 \mathrm{D}$ and $2 \mathrm{D}$ glioma models reveals increased HLA-E expression in 
3D models is associated with resistance to NK cell-mediated cytotoxicity. J. Proteome Res. 2014, 13, 2272-2281.

(14) Wu, Y.-M.; Tang, J.; Zhao, P.; Chen, Z.-N.; Jiang, J.-L. Morphological changes and molecular expressions of hepatocellular carcinoma cells in three-dimensional culture model. Exp. Mol. Pathol. 2009, 87, 133-140.

(15) Yue, X.; Lukowski, J. K.; Weaver, E. M.; Skube, S. B.; Hummon, A. B. Quantitative Proteomic and Phosphoproteomic Comparison of 2D and 3D Colon Cancer Cell Culture Models. J. Proteome Res. 2016, $15,4265-4276$

(16) Hughes, C. S.; Foehr, S.; Garfield, D. A.; Furlong, E. E.; Steinmetz, L. M.; Krijgsveld, J. Ultrasensitive proteome analysis using paramagnetic bead technology. Mol. Syst. Biol. 2014, 10, 757.

(17) Mootha, V. K.; Lindgren, C. M.; Eriksson, K.-F.; Subramanian, A.; Sihag, S.; Lehar, J.; Puigserver, P.; Carlsson, E.; Ridderstråle, M.; Laurila, E.; Houstis, N.; Daly, M. J.; Patterson, N.; Mesirov, J. P.; Golub, T. R.; Tamayo, P.; Spiegelman, B.; Lander, E. S.; Hirschhorn, J. N.; Altshuler, D.; Groop, L. C. PGC-1 $\alpha$-responsive genes involved in oxidative phosphorylation are coordinately downregulated in human diabetes. Nat. Genet. 2003, 34, 267-273.

(18) Subramanian, A.; Tamayo, P.; Mootha, V. K.; Mukherjee, S.; Ebert, B. L.; Gillette, M. A.; Paulovich, A.; Pomeroy, S. L.; Golub, T. R.; Lander, E. S.; Mesirov, J. P. Gene set enrichment analysis: a knowledge-based approach for interpreting genome-wide expression profiles. Proc. Natl. Acad. Sci. U.S.A. 2005, 102, 15545-15550.

(19) Chen, E. Y.; Tan, C. M.; Kou, Y.; Duan, Q.; Wang, Z.; Meirelles, G.; Clark, N. R.; Ma'ayan, A. Enrichr: interactive and collaborative HTML5 gene list enrichment analysis tool. BMC Bioinf. 2013, 14, 128.

(20) Kuleshov, M. V.; Jones, M. R.; Rouillard, A. D.; Fernandez, N. F.; Duan, Q.; Wang, Z.; Koplev, S.; Jenkins, S. L.; Jagodnik, K. M.; Lachmann, A.; McDermott, M. G.; Monteiro, C. D.; Gundersen, G. W.; Ma'ayan, A. Enrichr: a comprehensive gene set enrichment analysis web server 2016 update. Nucleic Acids Res. 2016, 44, W90W97.

(21) Perez-Riverol, Y.; Csordas, A.; Bai, J.; Bernal-Llinares, M.; Hewapathirana, S.; Kundu, D. J.; Inuganti, A.; Griss, J.; Mayer, G.; Eisenacher, M.; Pérez, E.; Uszkoreit, J.; Pfeuffer, J.; Sachsenberg, T.; Yllmaz, S.; Tiwary, S.; Cox, J.; Audain, E.; Walzer, M.; Jarnuczak, A. F.; Ternent, T.; Brazma, A.; Vizcaíno, J. A. The PRIDE database and related tools and resources in 2019: improving support for quantification data. Nucleic Acids Res. 2019, 47, D442-D450.

(22) Yue, X.; Lukowski, J. K.; Weaver, E. M.; Skube, S. B.; Hummon, A. B. Quantitative Proteomic and Phosphoproteomic Comparison of 2D and 3D Colon Cancer Cell Culture Models. J. Proteome Res. 2016, $15,4265-4276$.

(23) Mandujano-Tinoco, E. A.; Gallardo-Pérez, J. C.; MarínHernández, A.; Moreno-Sánchez, R.; Rodríguez-Enríquez, S. Antimitochondrial therapy in human breast cancer multi-cellular spheroids. Biochim. Biophys. Acta, Mol. Cell Res. 2013, 1833, 541-551. (24) Montero, J.; Morales, A.; Llacuna, L.; Lluis, J. M.; Terrones, O.; Basanez, G.; Antonsson, B.; Prieto, J.; Garcia-Ruiz, C.; Colell, A.; Fernandez-Checa, J. C. Mitochondrial Cholesterol Contributes to Chemotherapy Resistance in Hepatocellular Carcinoma. Cancer Res. 2008, 68, 5246-5256.

(25) Wang, C.; Li, P.; Xuan, J.; Zhu, C.; Liu, J.; Shan, L.; Du, Q.; Ren, Y.; Ye, J. Cholesterol Enhances Colorectal Cancer Progression via ROS Elevation and MAPK Signaling Pathway Activation. Cell. Physiol. Biochem. 2017, 42, 729-742.

(26) Jacobs, R. J.; Voorneveld, P. W.; Kodach, L. L.; Hardwick, J. C. Cholesterol metabolism and colorectal cancers. Curr. Opin. Pharmacol. 2012, 12, 690-695.

(27) Ghazalpour, A.; Bennett, B.; Petyuk, V. A.; Orozco, L.; Hagopian, R.; Mungrue, I. N.; Farber, C. R.; Sinsheimer, J.; Kang, H. M.; Furlotte, N.; Park, C. C.; Wen, P. Z.; Brewer, H.; Weitz, K.; Camp, D. G.; Pan, C.; Yordanova, R.; Neuhaus, I.; Tilford, C.; Siemers, N.; Gargalovic, P.; Eskin, E.; Kirchgessner, T.; Smith, D. J.; Smith, R. D.; Lusis, A. J. Comparative analysis of proteome and transcriptome variation in mouse. PLoS Genet. 2011, 7, No. e1001393.
(28) Pereira, S. F. F.; Gonzalez, R. L.; Dworkin, J. Protein synthesis during cellular quiescence is inhibited by phosphorylation of a translational elongation factor. Proc. Natl. Acad. Sci. U.S.A. 2015, 112, E3274-E3281.

(29) Zhang, X.; Fryknäs, M.; Hernlund, E.; Fayad, W.; De Milito, A.; Olofsson, M. H.; Gogvadze, V.; Dang, L.; Påhlman, S.; Schughart, L. A. K.; Rickardson, L.; D’Arcy, P.; Gullbo, J.; Nygren, P.; Larsson, R.; Linder, S. Induction of mitochondrial dysfunction as a strategy for targeting tumour cells in metabolically compromised microenvironments. Nat. Commun. 2014, 5, 3295.

(30) Pandyra, A.; Mullen, P. J.; Kalkat, M.; Yu, R.; Pong, J. T.; Li, Z.; Trudel, S.; Lang, K. S.; Minden, M. D.; Schimmer, A. D.; Penn, L. Z. Immediate Utility of Two Approved Agents to Target Both the Metabolic Mevalonate Pathway and Its Restorative Feedback Loop. Cancer Res. 2014, 74, 4772-4782.

(31) Shen, S.-L.; Qiu, F.-H.; Dayarathna, T. K.; Wu, J.; Kuang, M.; Li, S. S.-C.; Peng, B.-G.; Nie, J. Identification of Dermcidin as a novel binding protein of Nck1 and characterization of its role in promoting cell migration. Biochim. Biophys. Acta, Mol. Basis Dis. 2011, 1812, 703-710.

(32) Mikhaylova, M.; Schumacher, A.; Borutzki, C.; Neumann, J. R.; Macharadze, T.; El-Mousleh, T.; Wahle, P.; Zenclussen, A. C.; Kreutz, M. R. Analysis of Y-P30/Dermcidin expression and properties of the Y-P30 peptide. BMC Res. Notes 2014, 7, 400.

(33) Moore, C. E. J.; Wang, X.; Xie, J.; Pickford, J.; Barron, J.; Regufe da Mota, S.; Versele, M.; Proud, C. G. Elongation factor 2 kinase promotes cell survival by inhibiting protein synthesis without inducing autophagy. Cell. Signalling 2016, 28, 284-293.

(34) Bekker-Jensen, D. B.; Kelstrup, C. D.; Batth, T. S.; Larsen, S. C.; Haldrup, C.; Bramsen, J. B.; Sørensen, K. D.; Høyer, S.; Ørntoft, T. F.; Andersen, C. L.; Nielsen, M. L.; Olsen, J. V. An Optimized Shotgun Strategy for the Rapid Generation of Comprehensive Human Proteomes. Cell Syst. 2017, 4, 587-599.e4.

(35) LaBonia, G. J.; Ludwig, K. R.; Mousseau, C. B.; Hummon, A. B. iTRAQ Quantitative Proteomic Profiling and MALDI-MSI of Colon Cancer Spheroids Treated with Combination Chemotherapies in a 3D Printed Fluidic Device. Anal. Chem. 2018, 90, 1423-1430. 